\title{
Dual drug-loaded PLA nanoparticles bypassing drug resistance for improved leukemia therapy
}

\author{
Liuliu Zhang • Huayun Zhu • Yu Gu • Xiaohua Wang • \\ Pingping Wu
}

Received: 1 May 2018 / Accepted: 21 November 2018 /Published online: 22 April 2019

(C) The Author(s) 2019

\begin{abstract}
Drug resistance is still a bottle-neck hindering successful chemotherapy in leukemia treatment. Nanocarriers have emerged as promising candidates to circumvent drug resistance and discover potent drug combinations. Here, we showed that co-encapsulation of daunorubicin (DNR) and glycyrrhizic acid (GA) in polylactic acid (PLA) nanoparticles effectively bypassed drug resistance and remarkably inhibited the growth of drug-resistant leukemia cells. The quantification of intracellular drug found that the encapsulation effectively increased drug uptake in the resistant K562/ A02 cells. Modification of P-glycoprotein antibody on
\end{abstract}

Liuliu Zhang and Huayun Zhu contributed equally to this work.

\section{Zhang $\cdot$ H. Zhu $\cdot$ X. Wang $\cdot$ P. Wu $(\bowtie)$}

Department of Medical Oncology, Jiangsu Cancer Hospital \& Jiangsu Institute of Cancer Research \& The Affiliated Cancer Hospital of Nanjing Medical University, Nanjing 210009, China e-mail: wupingpingnano@ sina.com

\section{Zhang \\ e-mail: zhangliuliu212@163.com}

H. Zhu

e-mail: 304311140@qq.com

X. Wang

e-mail: wangxiaohua80@ hotmail.com

Y. Gu

Department of General Medicine, Jiangsu Province Traditional Chinese Medicine Hospital, Nanjing 210029, China

e-mail: guyugg@hotmail.com nanoparticles further enhanced drug accumulation in the leukemia cells, which was also confirmed by fluorescent microscopy imaging. More importantly, loaded in PLA nanoparticles, DNR and GA exerted an excellent synergistic effect, leading to significantly improved cell inhibition compared to the treatments without nanocarriers and those with a single drug. Correlative mechanism study revealed that the drug delivery system drastically enhanced cell apoptosis by regulating apoptotic genes but did not influence MDR1 expression. The present study proposes a versatile strategy to improve the therapeutic efficacy against drug-resistant leukemia and inspires the discovery of more potent drug combinations for enhanced leukemia therapy.

Keywords Multidrug resistance $\cdot$ Cancer chemotherapy $\cdot$ P-glycoprotein $\cdot$ Nanocapsules $\cdot$ Targeted drug delivery $\cdot$ Natural products

\section{Introduction}

Leukemia is a life-threatening cancer featured by fast proliferation of abnormal white blood cells in the bone marrow, which interferes with the production of normal blood cells and deteriorates rapidly (Kihara et al. 2014; Picaud et al. 2015; Nishida et al. 2015). Although clinical strategies against leukemia have gained rapid development in recent years, multidrug resistance still hinders effective therapy, especially for drug-resistant cancer. 
Prolonged drug exposure or larger doses are often used to combat resistant cancer cells, but subsequently developed leukemia may be induced, which is a detrimental consequence after chemotherapy (Whitlock et al. 1991; Bishop 1997). Moreover, due to multidrug resistance, the patients achieving partial or complete remission after conventional treatments often suffer from tumor recurrence with low disease-free survival rate (Holohan et al. 2013; Li et al. 2014; Zhang et al. 2014, 2015a, b). On the other hand, conventional chemotherapy often cause various unpleasant side effects due to non-specific drug distribution that poses unavoidable toxicity to normal tissues (Döhner et al. 2015; Fox et al. 2017; Stauss and Morris 2013). Therefore, it is particularly attractive to precisely deliver drugs to cancer cells, overcome drug resistance, and relieve the toxicity of chemotherapy.

Nanocarriers with distinct advantages and multiple functionalities can serve as potential candidates to address the above issues (Chen et al. 2017; Zhang et al. $2015 \mathrm{a}, \mathrm{b})$. The encapsulation of chemotherapeutic agents, such as doxorubicin and daunorubicin, in nanocarriers facilitates the bypass of efflux-pump in resistant cells (Meng et al. 2013; Zhu et al. 2014; Kirtane et al. 2013; Loos et al. 2014). In addition, nanocarriers have been proved effective to reduce side effects by improving the pharmacokinetic property and increasing drug concentration in tumor (Wang et al. 2013; Rochani et al. 2016; Zhao and Zhu 2016; Singh et al. 2010). However, few of the reported nanosystems have demonstrated a potent effect in drug-resistant myeloid leukemia that responds poorly to first-line chemotherapy. To further improve the therapeutic efficacy of nano-drugs in leukemia, the co-encapsulation of different chemo-drugs is particularly appealing.

As a broad-spectrum anticancer drug, daunorubicin (DNR) is remarkably effective in both solid tumors and leukemia by disrupting DNA replication. However, drug resistance and its cardiotoxicity have substantially limited its clinical application (Sheng et al. 2015; Halley et al. 2016). Glycyrrhizic acid (GA) is a natural product extracted from the root of the plant Glyccyrhiza glabra (liquorice) with multiple therapeutic effects (Csuk 2014; Cai et al. 2016). It was found to induce apoptosis in different types of promyelotic leukemia (Hibasami et al. 2005; Thirugnanam et al. 2008). Different from DNR, GA suppresses the proliferation of leukemia cells mainly by interfering with the AKT signaling pathway (He et al. 2015). Therefore, a synergistic effect may be achieved through the combination of DNR with GA.
Nonetheless, to the best of our knowledge, the combination of DNR and GA in one nanocarrier remains barely explored in leukemia therapy.

Among the numerous categories of nanocarriers for drug delivery, including liposomes, polymeric micelles, dendrimers, and inorganic nanoparticles (Mura et al. 2013), polylactic acid (PLA) has attracted much attention due to its chemical versatility, biodegradability, and biocompatibility, which are important properties for further development (Danafar et al. 2014; Cui et al. 2013). For the delivery of specific drugs, it is also necessary to compare the drug loading properties of different nanoparticles to select the optimal carrier. In this study, PLA/ polyvinyl alcohol (PVA) nanoparticles were encapsulated with DNR and GA and further functionalized with Pglycoprotein (P-gp) antibody for targeted drug delivery in resistant leukemia cells. The nanosystem was constructed and optimized with detailed evaluation of encapsulation efficiency (EE) and drug loading capacity (DL). The comparison of EE and DL with chitosan and albumin nanoparticles was also performed. Then, circumvent of efflux-pump and the improved drug uptake by antibody modification were proved by fluorescence microscopy and HPLC quantification. Afterwards, we evaluated if the drug combination could substantially improve the therapeutic efficacy against MDR leukemia cells. Further, we revealed the underlying molecular mechanism by which the nanosystem circumvented drug resistance. To provide basis for future in vivo study, the toxicity of the nanoconstruct was evaluated on healthy mice. Altogether, this study has provided solid evidence for the combined application of DNR and GA in one simple nanosystem, inspiring the development of nano-medicine for more effective therapy against drug-resistant leukemia.

\section{Materials and methods}

Preparation of drug-carried nanocapsules

The solvent evaporation method was used to prepare the nanocapsules, which carried both GA and DNR. The total mass of nanocapsules were fixed to $1 \mathrm{~g}$. Briefly, PLA (350 mg) and PVA (150 mg) were dissolved in chloroform by $5 \mathrm{~min}$ ultrasound. GA $(250 \mathrm{mg})$ and DNR $(250 \mathrm{mg})$ were then added into the solution for another $5 \mathrm{~min}$ ultrasound. Afterwards, the suspension was slowly dropped into the PVA aqueous solution and 
stirred constantly for $2 \mathrm{~h}$, of which the DNR and GA were saturated. Third, the milky white product, "water in oil in water" $(\mathrm{W} / \mathrm{O} / \mathrm{W})$ compound emulsion, was formed after 10,000 rpm centrifugation for $1 \mathrm{~h}$. Rotary evaporator was then used to vaporize the chloroform completely. Finally, the microcapsules were washed with distilled water for 4-5 times at room temperature, and dried with a vacuum dryer for $24 \mathrm{~h}$. To conjugate $\mathrm{P}-$ g Ab on the nanoparticles, $25 \%$ glutaraldehyde solution was added into the microcapsules at room temperature. After 10 min cross-linking, the excessive glutaraldehyde was removed with physiological saline by dialysis for 4 h. Five micrograms P-g Ab was then added into the solution with magnetic stirring and reacted overnight. A laser diffraction micrometer particle size measuring instrument (Master Sizer) was adapted to measure the nanoparticle diameter with the function of the Spherical multimodal model, and the structure of nanocapsules was observed by transmission electron microscope.

For the preparation of chitosan nanoparticles, chitosan $(1.0 \mathrm{~g}, 50 \mathrm{kDa})$ was dissolved and then dispersed in methanol with fierce stirring. Succinic anhydride acetone $\left(0.18 \mathrm{~g} \mathrm{~mL}^{-1}\right)$ was reacted with chitosan for $36 \mathrm{~h}$ and the mixture was neutralized by $1 \mathrm{M} \mathrm{NaOH}$. After lyophilization, the products were re-dispersed in $1 \%$ acetic acid and reacted with $0.5 \mathrm{~g}$ octaldehyde for $6 \mathrm{~h}$. Then, $0.2 \mathrm{~g} / \mathrm{mL} \mathrm{NaBH}_{4}$ was slowly added and reacted overnight. The reaction was stopped by $1 \mathrm{M} \mathrm{NaOH}$, and the final products were obtained after dialyzation and lyophilization. BSA nanoparticles of $100 \mathrm{~nm}$ diameter were prepared by adding $1 \%$ BSA in distilled water with a $\mathrm{pH}$ value of 9. The solution was then stirred at $500 \mathrm{rpm}$ for $5 \mathrm{~h}$, and the final products were obtained after dialyzation and lyophilization.

Optimization of drug loading capacity and encapsulation efficiency

On the basis of a single factor, orthogonal design was used to evaluate the effects of different factors on the loading efficiency and encapsulation capacity. The factors investigated were emulsifier, polyvinyl alcohol (PVA) concentration, poly lactic acid (PLA) concentration, drug carrier ratio, dispersion phase (oil phase), and volume ratio of the continuous phase (aqueous phase), respectively. Parallel experiments were conducted at three levels for each factor, and the encapsulation efficiency EE\% and drug loading DL\% were evaluated by HPLC. The HPLC conditions to quantify DNR and GA in the complex are as follows: the complex suspensions were dissolved in $3 \mathrm{~mL} 0.1 \%$ mixed solution (TFA/ $\mathrm{MeOH}=3: 1$ ). Then, DNR and GA were quantified with the Agilent 1100 system and reversed phase C18 column. For DNR, the mobile phase was $0.1 \%$ mixed solution of TFA/MeOH $=3: 1$, and acetonitrile. The DNR concentration was determined at $480 \mathrm{~nm}$ under $1 \mathrm{~mL} / \mathrm{min}$ flow rate. For GA, gradient elution was used containing $0.05 \%$ trifluoroacetic acid with $0.8 \mathrm{~mL} / \mathrm{min}$ flow rate. GA concentration was measured at $254 \mathrm{~nm}$ with $15 \mathrm{~min}$ retention time.

\section{Cell culture}

The multidrug-resistant leukemia cell K562/A02 was purchased from the Shanghai Institute of Cell Biology, Chinese Academy of Sciences (Shanghai, China). K562/A02 cells were cultured in RPMI 1640 (Gibco, USA) supplemented with $10 \%$ FBS, $100 \mathrm{mg} / \mathrm{mL}$ streptomycin, and $100 \mathrm{IU} / \mathrm{ml}$ penicillin in $5 \% \mathrm{CO}_{2}$ atmosphere at $37^{\circ} \mathrm{C}$.

Fluorescence microscopy

K562/A02 cells were divided into two groups and incubated with the dual drug-loaded PVA/PLA nanoparticles with and without P-glycoprotein, respectively. Drug concentrations were 1 and $10 \mu \mathrm{M}$ for DNR and GA, respectively. The cells were subjected to fluorescent microscopy and observed for $10 \mathrm{~h}$ to evaluate the cellular uptake of the nanoparticles.

\section{Cell proliferation assay}

Cell proliferation was analyzed using the Cell Proliferation Reagent Kit 1(MTT) (Sigma, USA). In total, K562/A02cells were seeded in 96-well plates at $5 \times$ $10^{3}$ per well and cultured for $24 \mathrm{~h}$. MTT solution was added into each well for $4 \mathrm{~h}$. After removing the medium, the absorbance was measured using a Microplate Reader (Bio-Rad, Hercules, CA, USA) at $490 \mathrm{~nm}$. Each experiment was conducted in triplicate.

Cell apoptosis analysis

Cell apoptosis was performed using the Annexin V Apoptosis Detection kit I (BD Biosciences, USA). After incubation for $24 \mathrm{~h}$, cells were digested with trypsin and washed in cold PBS twice. Subsequently, $500 \mu \mathrm{L}$ buffer, 
$5 \mu \mathrm{L}$ FITC annexin $\mathrm{V}$, and $5 \mu \mathrm{L}$ propidium iodide (PI) were added. Finally, apoptosis was assessed by flow cytometry (FACScan, BD Biosciences).

qRT-PCR analysis

Total RNA was extracted from tissue samples and cultured cells using the Trizol reagent (Invitrogen) according to the manufacturer's instructions. Total RNA was reversed transcribed into cDNA using the TaqMan microRNA Reverse Transcription Kit (Applied Biosystems, USA). The qRT-PCR reactions were performed using the Fast Start Universal SYBR Green Master (Rox) (Roche Applied Science) on a 7500 Real-time system (Applied Biosystems, USA) according to the manufacturer's protocols. The levels of miR10a and Tiam1 were normalized by U6 and $\beta$-actin, respectively. Primer pairs used in this study were the following:

\section{Homo-Bcl-2: 5'-GGTGGGGTCATGTGTGTGG- 3' (forward) \\ Homo-Bcl-2: 5'-CGGTTCAGGTACTCAGTCAT CC-3' (reverse) \\ Homo-Bax: 5'-CCCGAGAGGTCTTTTTCCGA G-3' (forward) \\ Homo-Bax: 5'-CCAGCCCATGATGGTTCTGAT- $3^{\prime}$ (reverse)}

The qRT-PCR reactions were performed in triplicate and included no-template controls.

\section{Western blot}

Total proteins were extracted from cells using the RIPA lysis buffer (50 mM Tris-HCl, pH 7.4, $150 \mathrm{mM} \mathrm{NaCl}$, $0.2 \%$ SDS, $1 \%$ Triton X-100, 2 mM EDTA) for $30 \mathrm{~min}$ on ice. The supernatant was collected by centrifuging at $12,000 \mathrm{rpm}, 10 \mathrm{~min}$. After the loading buffer was added, the mixtures were treated at $100^{\circ} \mathrm{C}$ for $5 \mathrm{~min}$ to denature the protein. Protein concentration was determined by the BCA Quantification Kit (Beyotime, Beijing, China). The samples $(30 \mathrm{mg})$ were separated on $10 \%$ SDSPAGE and blotted onto a PVDF membrane. The membranes were blocked with 5\% no-fat milk $(w / v)$ at room temperature for $1 \mathrm{~h}$ and incubated with primary antibodies overnight at $4{ }^{\circ} \mathrm{C}$ : rabbit anti-human GAPDH antibody (1: 2000, Cell Signaling Technology, Beverly, MA, USA), rabbit anti-human Bax antibody (1: 2000,
Cell Signaling Technology, Beverly, MA, USA), and rabbit anti-human Bcl-2 antibody (1: 2000, Cell Signaling Technology, Beverly, MA, USA). After incubation, the membranes were washed with PBST three times again, and then incubated with a horseradish peroxidase (HRP)-conjugated secondary antibody (Boster Biotechnology, Wuhan, China) at room temperature for $2 \mathrm{~h}$. The immuno-reactivity was visualized using the ECL Western blotting kit (Beyotime, Beijing, China) according to the manufacturer's protocols. GAPDH was employed as a loading control.

In vivo toxicity assay

Balb/c nude mice of 6 weeks old were purchased from Qing Long Shan company, Nanjing, China. Six mice were randomly divided into a control group and an experiment group with three mice in each group. Mice in the experiment group were injected with nanoparticles ( $1 \mathrm{mg} / \mathrm{kg}$ DNR and $1 \mathrm{mg} / \mathrm{kg} \mathrm{GA}$ ) every 2 days for 15 days. The free-drugs group was treated with $1 \mathrm{mg} / \mathrm{kg}$ DNR and $1 \mathrm{mg} / \mathrm{kg}$ GA without nano-encapsulation. The control group was injected with saline at the same time points. Hematoxylin-eosin (HE) staining was performed for the major organs after treatments. Animal experiments were carried out in accordance with Guidelines for the Care and Use of Laboratory Animals in Jiangsu Cancer Hospital.

Statistical analysis

The experiments in this study were performed for three times and data were presented as mean $\pm \mathrm{SD}$. One-way ANOVA with Tukey-Kramer multiple comparison post hoc tests were used for statistical analysis. $* P<0.05$ and $* * P \leq 0.01$ were used to indicate that the difference is statistically significant.

\section{Results and discussion}

Preparation and characterization of DNR/GA-loaded PVA/PLA nanoparticles

PVA/PLA nanoparticles were successfully prepared using the solvent evaporation method. DLS data showed that the mean diameter of the nanoparticles was about $90 \mathrm{~nm}$, which increased to approximately $110 \mathrm{~nm}$ after P-p-glycoprotein antibody modification (Fig. 1a, b). 
Also, Zeta potential shifted from -57 to $-20 \mathrm{mV}$ after antibody attachment (Fig. 1c). The narrow size distribution evidenced the overall homogenous property of our products. TEM images of the original (left) and antibody cross-linked (right) particles further confirmed the uniform distribution with spherical morphology, well consistent with the DLS data (Fig. 1d). To achieve an optimal drug loading efficiency and encapsulation capacity, the ingredients of the nanocarrier were carefully adjusted. The proportion of the main components, including the oil phase (PLA, poly lactic acid), water phase (DNR/GA), and surfactant phase (PVA, polyvinyl alcohol), was investigated. The results of the average EE\% (encapsulation efficiency) and DL\% (drug loadings) were used to provide a comprehensive evaluation of these factors (Fig. 2a). The optimal formulation was selected with $50 \%$ water, $40 \%$ PLA, and $10 \%$ PVA, achieving EE\% of 68.1 and DL\% of 1.29, respectively, a)

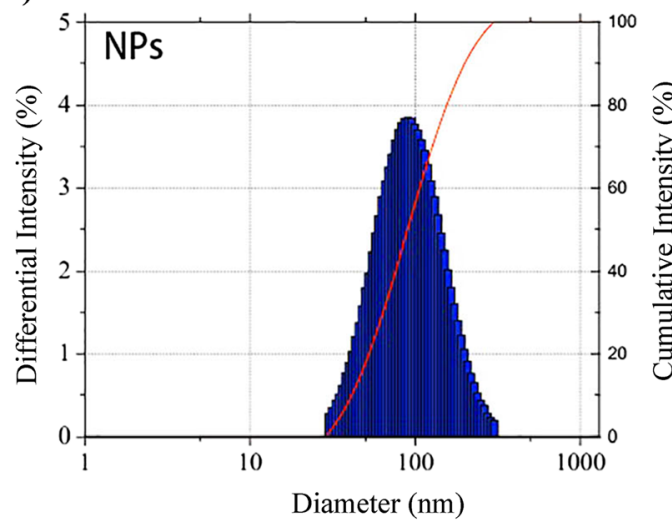

c)

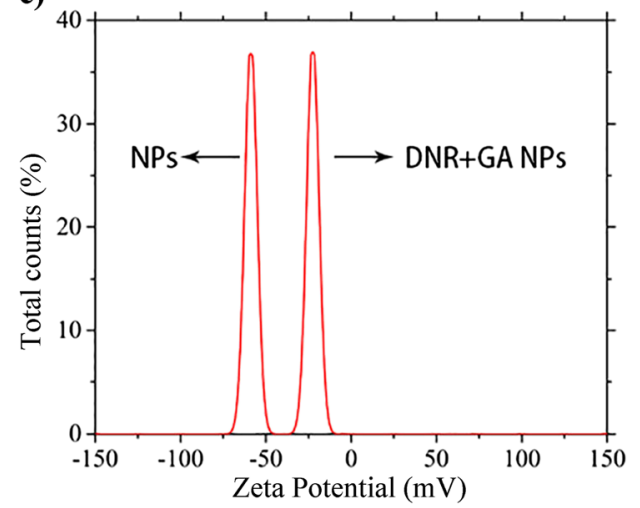

Fig. 1 Characterization of the prepared dual drug-loaded PVA/ PLA nanoparticles. a, b Dynamic light scattering (DLS) analysis of the diameter of the nanoparticles before and after p-glycoprotein antibody modification. $\mathbf{c}$ Changes of ZETA potential before and for DNR. Similarly, this formulation also resulted in favorable EE\% (3) and DL\% (1.13) for GA. Moreover, the EE and DL of our prepared nanoparticles were compared with other commonly used carriers including chitosan and albumin (Fig. 2b, c). The PLA nanoparticles demonstrated higher EE and DL for DNR and GA, probably because PLA nanoparticles were more appropriate to load hydrophilic drugs compared with chitosan and albumin. In vitro release of the loaded DNR and GA were tested at $37{ }^{\circ} \mathrm{C}$ in PBS solutions at a $\mathrm{pH}$ value of 7.4 , which represented the normal physiological environments. The HPLC peaks and corresponding standard curves used for the calculation were shown in Fig. 3a. GA and DNR showed similar drug release profiles during the 96-h examination, which showed more than $90 \%$ release at $48 \mathrm{~h}$ and remained nearly stable afterwards (Fig. 3b).

b)

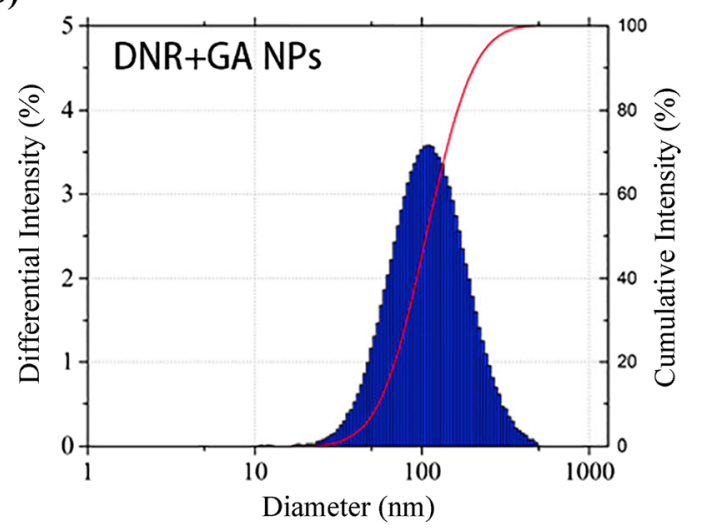

d)

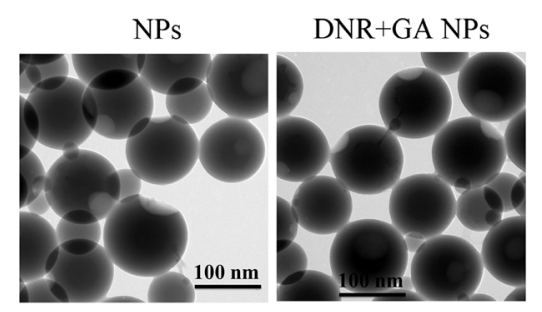

after antibody modification. d TEM images of the dual drugloaded PVA/PLA nanoparticles before and after antibody modification 
a)

\begin{tabular}{|c|c|c|c|c|c|}
\hline \multirow{2}{*}{$\begin{array}{c}\text { Formulation } \\
\text { Numbers }\end{array}$} & \multicolumn{3}{|c|}{ Formulation Components } & \multirow{2}{*}{ EE\% } & \multirow{2}{*}{ DL\% } \\
\cline { 2 - 4 } & Water/\% & Oil(PLA)/\% & PVA/\% & & \\
\hline 1 & 80 & 10 & 10 & $59.1 \pm 5.23$ & $1.36 \pm 0.04$ \\
\hline 2 & 20 & 70 & 10 & $65.5 \pm 2.10$ & $1.29 \pm 0.19$ \\
\hline 3 & 20 & 10 & 70 & $76.4 \pm 2.54$ & $1.18 \pm 0.01$ \\
\hline 4 & 40 & 30 & 30 & $65.9 \pm 0.36$ & $1.24 \pm 0.07$ \\
\hline 5 & 50 & 40 & 10 & $68.1 \pm 2.56$ & $1.29 \pm 0.17$ \\
\hline 6 & 50 & 10 & 40 & $53.0 \pm 1.11$ & $1.45 \pm 0.24$ \\
\hline 7 & 20 & 40 & 40 & $66.6 \pm 0.45$ & $0.84 \pm 0.12$ \\
\hline
\end{tabular}

b)

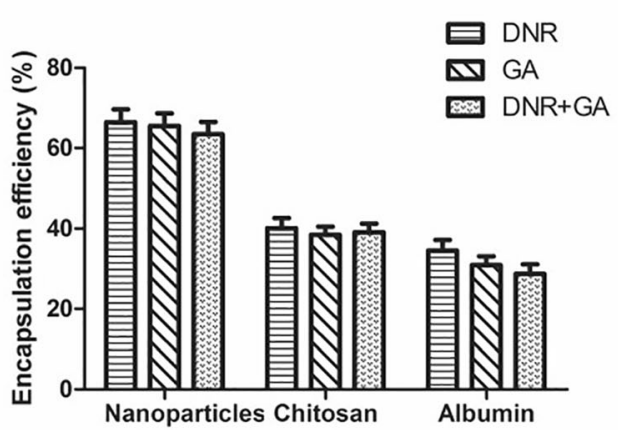

Fig. 2 Evaluation and optimization of drug loading capacity and encapsulation efficiency. a Changes of drug loading capacity and encapsulation efficiency with different formulation components. $\mathbf{b}$ Comparison of encapsulation efficiency of PVA/PLA

Cellular uptake of DNR/GA-loaded PVA/PLA nanoparticles

The overexpression of P-glycoprotein largely contributes to multidrug resistance (MDR) of cancer cells, which can also be used to differentiate malignant cells from normal ones. Thus, targeting P-glycoprotein has been extensively explored for specific drug delivery to MDR cells ( $\mathrm{Li}$ et al. 2010). To improve the precision of the nanoparticles to kill leukemia cells, the Pglycoprotein antibody was modified on the particle surface. In this experiment, K562/A02 cells were incubated with the nanostructure or free drug for different time periods and then subjected to fluorescent microscopy. As a multidrug-resistant leukemia cell line, K562/A02 is derived from the parent $\mathrm{K} 562$ cells that are sensitive to chemotherapy. Fluorescent microscopy found that DNR encapsulated in PLA nanoparticles gradually accumulated in the drug-resistant cells during the 10-h monitoring, as illustrated by the increased fluorescence. Therefore, PLA-mediated drug delivery could overcome the c)

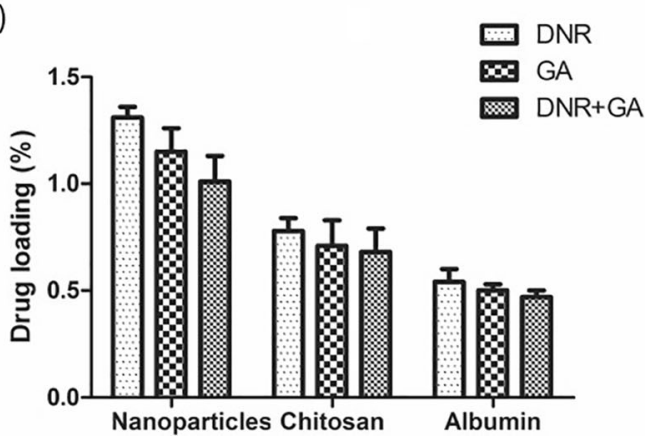

nanoparticles with conventional carriers of chitosan and albumin. c Comparison of drug loading capacity of PVA/PLA nanoparticles with conventional carriers of chitosan and albumin

expulsion of DNR from drug-resistant cancer cells. For antibody conjugated nanoparticles, obvious DNR fluorescence was soon observed $1 \mathrm{~h}$ after incubation, which increased sustainably and peaked at $10 \mathrm{~h}$, which was significantly stronger than that without antibody modification (Fig. 4). It was then believed that the Pglycoprotein antibody could further improve intracellular drug distribution by active targeting.

Further, we quantified the intracellular drug amount at different time points for various treatments in both K562 and K562/A02 cells. The percentages of cellular uptake were calculated by comparing the originally added drug and the drug remained in the supernatants using HPLC. As shown in Fig. 4b, the amount of free DNR gradually increased during the 10 -h incubation time in parental $\mathrm{K} 562$ cells,to about $1.3 \%$ of the total incubated drug. For the resistant K562/A02 cells, intracellular DNR decreased from $0.25 \%$ at $1 \mathrm{~h}$ to about $0.1 \%$ at $10 \mathrm{~h}$. For the PLA-encapsulated DNR, the uptake percentage in both K562 and K562/A02 cells increased to about $1 \%$ in $10 \mathrm{~h}$, indicating that 

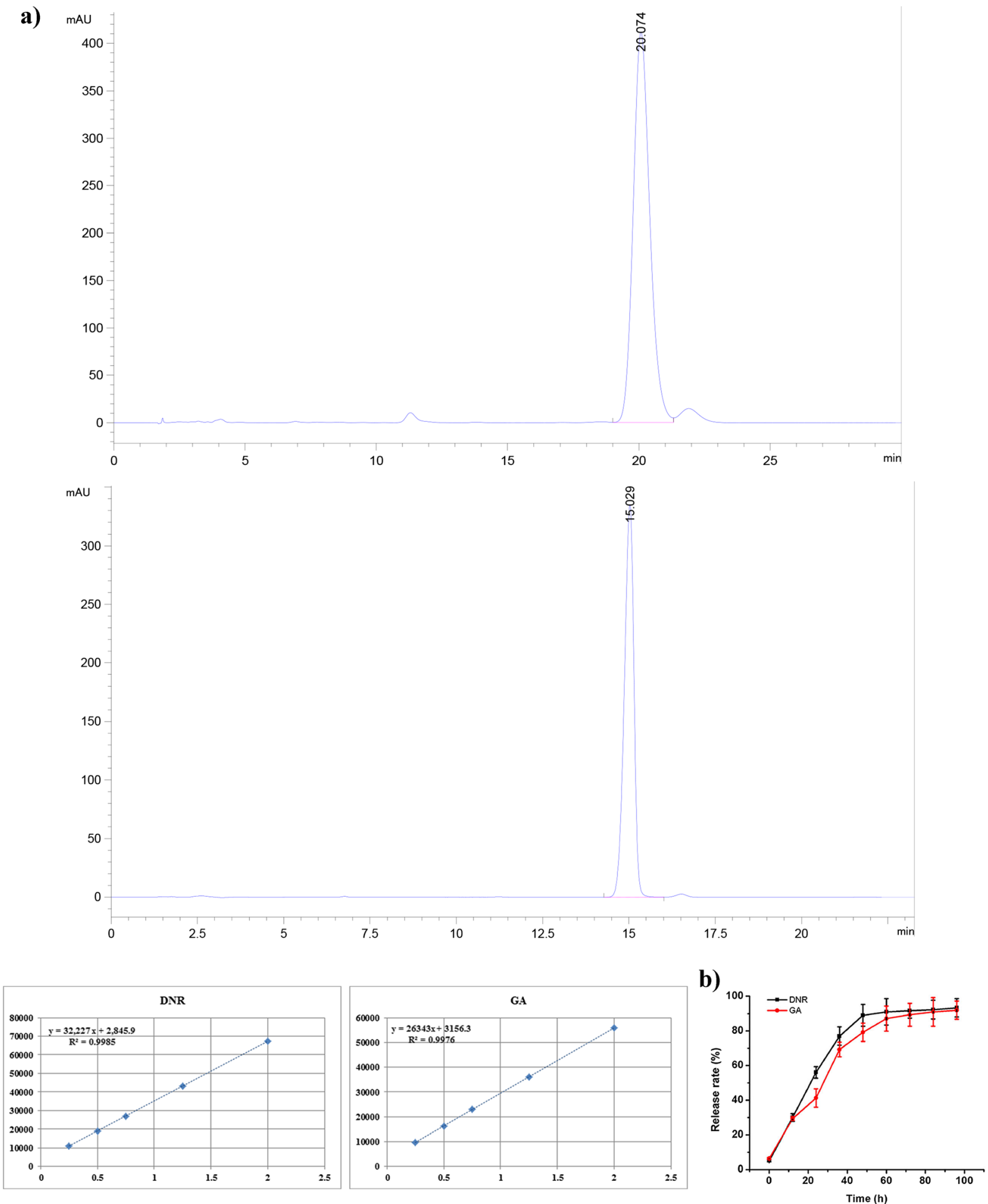

Fig. 3 a HPLC peaks of DNR and GA from instrument and corresponding standard curves. b Release profiles of DNR and GA from the PVA/PLA nanoparticles in PBS solution within $96 \mathrm{~h}$ 
a)

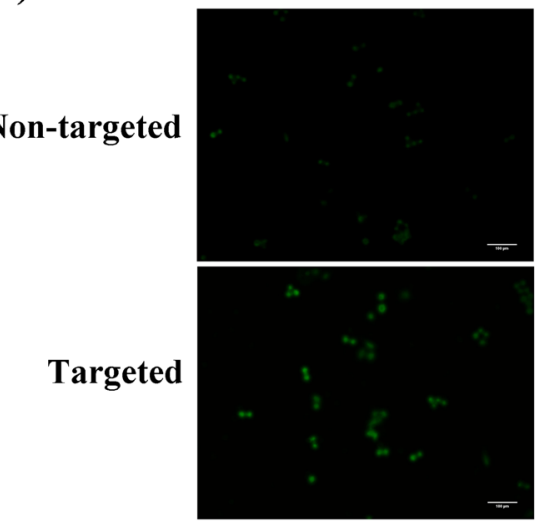

b)

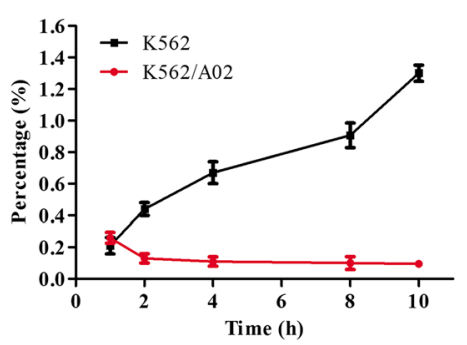

Fig. 4 a Fluorescent imaging of cellular uptake of the drug-loaded nanocarriers with and without P-glycoprotein antibody modification at $1,4,8$, and $10 \mathrm{~h}$ after incubation. $\mathbf{b}$ Quantification of the

the uptake of nanoparticles was not compromised by drug resistance. Finally, the antibody conjugated nanoparticles demonstrated the most abundant drug
PLA-DNR

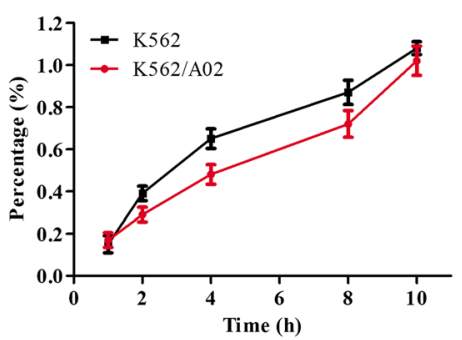

8 h

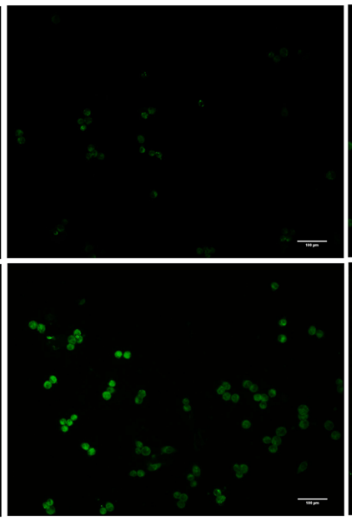

$10 \mathrm{~h}$

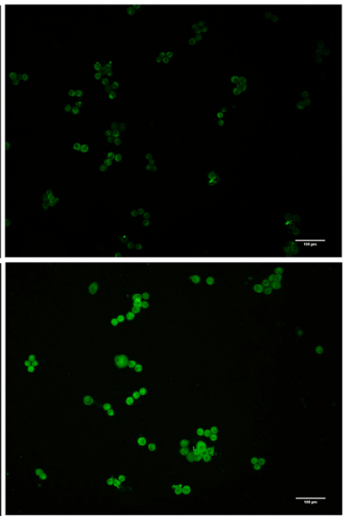

Ab-PLA-DNR

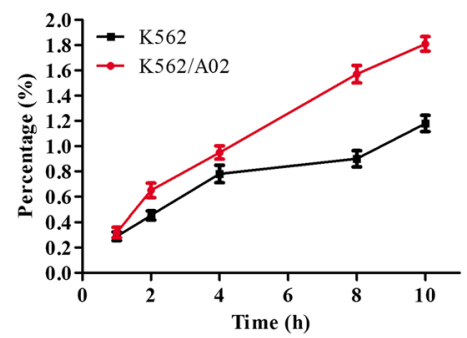

intracellular drug amount at different time points for various treatments in K562 and K562/A02 cells. Scale bar $=100 \mu \mathrm{m}$

accumulation in the drug-resistant K562/A02 cell line, reaching $1.8 \%$ of the originally added drug. These data strongly supported the ability of the
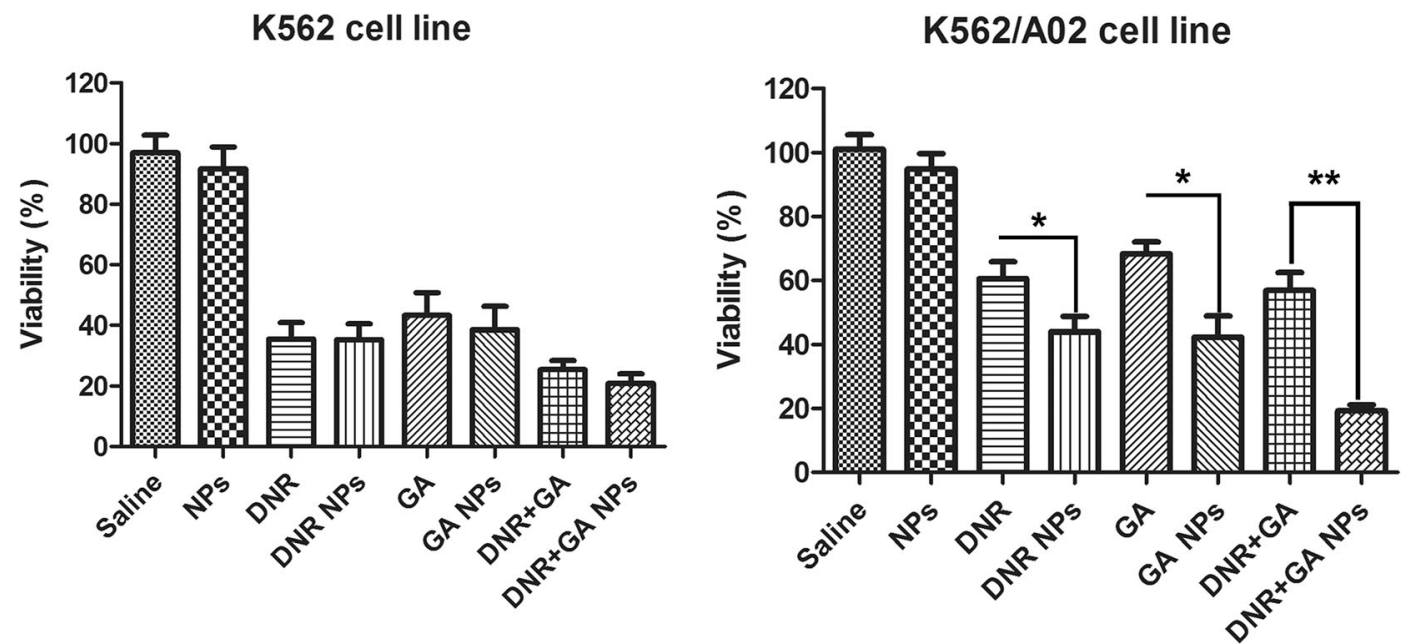

Fig. 5 MTT results of the efficacy of different treatments on K562 and K562/A02 cells. Data were presented as mean \pm SD $(n=3)$. $* * P<0.01, * P<0.05$ 

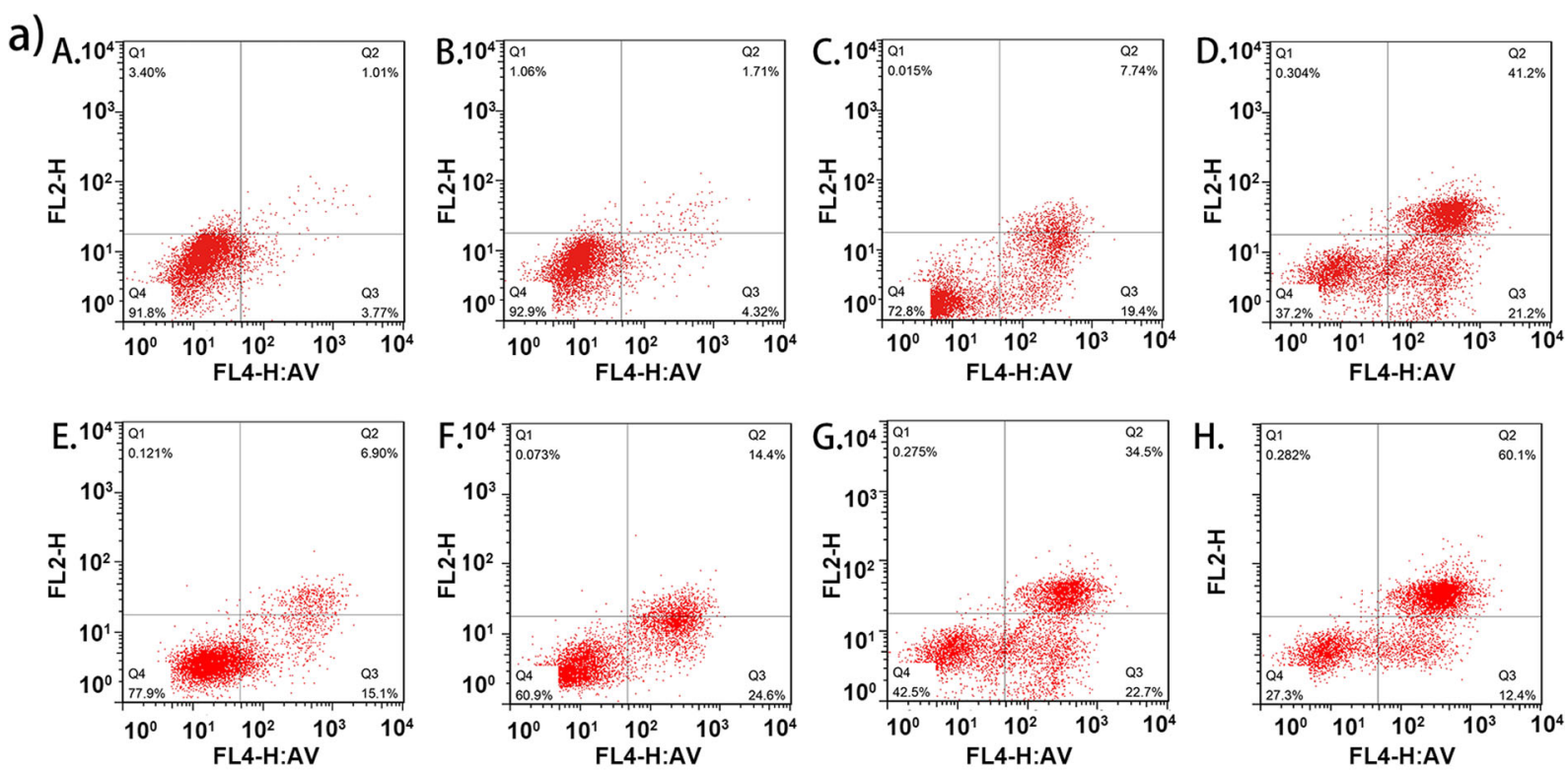

b)

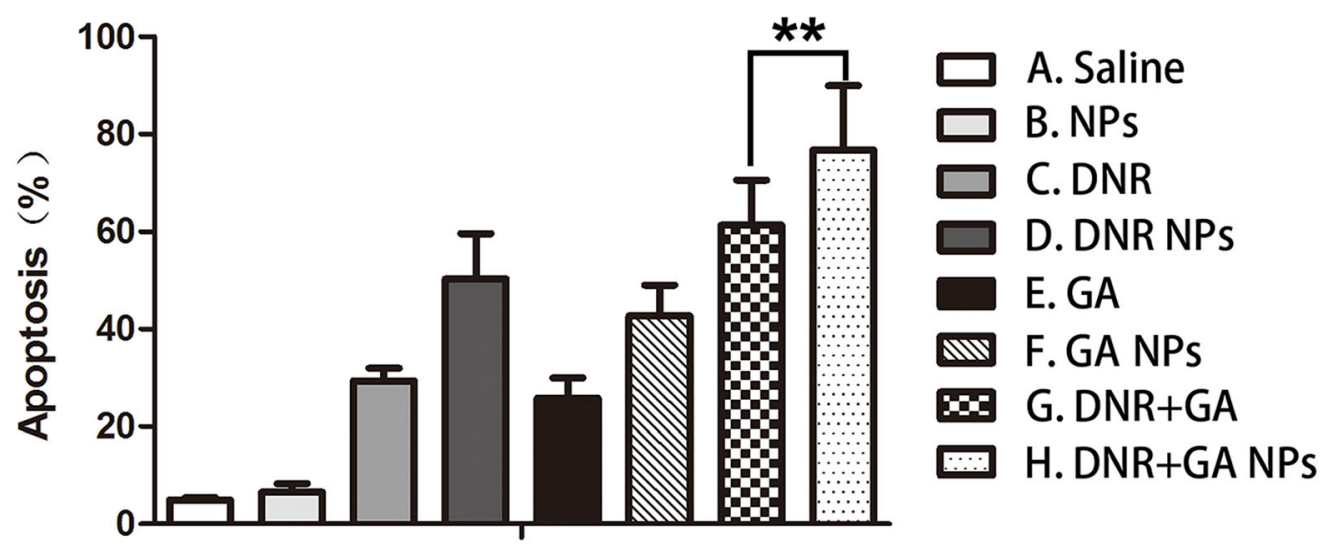

Fig. 6 Flow cytometry analysis of the apoptosis of the K562/A02 cells with different treatments. Data were presented as mean \pm SD $(n=3)$. $* * P<0.01$

drug-loaded PLA nanoparticles to circumvent drug resistance and the effectiveness of P-glycoprotein antibody modification on improving drug internalization.

Therapeutic efficacy of DNR/GA-loaded PVA/PLA nanoparticles

Given the nanosystem was readily internalized by K562/A02 cells, it was speculated that the drug resistance could be circumvented. We also used the parental K562 cell line without drug resistance as control. In this experiment, the cells were divided into 8 groups to comprehensively evaluate the therapeutic efficacy of different treatments after $24 \mathrm{~h}$ incubation. The concentrations of the drugs were 1 and $10 \mu \mathrm{M}$ for DNR and GA, respectively. As shown in Fig. 5, the nanoparticles without drug loading showed favorable biocompatibility in both K562 and K562/A02 cells, demonstrating comparable cell viability to the saline control. For the non-resistant K562 cells, free DNR treatment effectively inhibited cell proliferation, eradicating more than $60 \%$ of cells after $24 \mathrm{~h}$ incubation. Free GA also potently suppressed cell growth. The combined use of DNR and GA induced a more pronounced cell killing effect. It was observed that K562 cells were sensitive to both drugs, and the nanoparticle delivery did not significantly 
a)
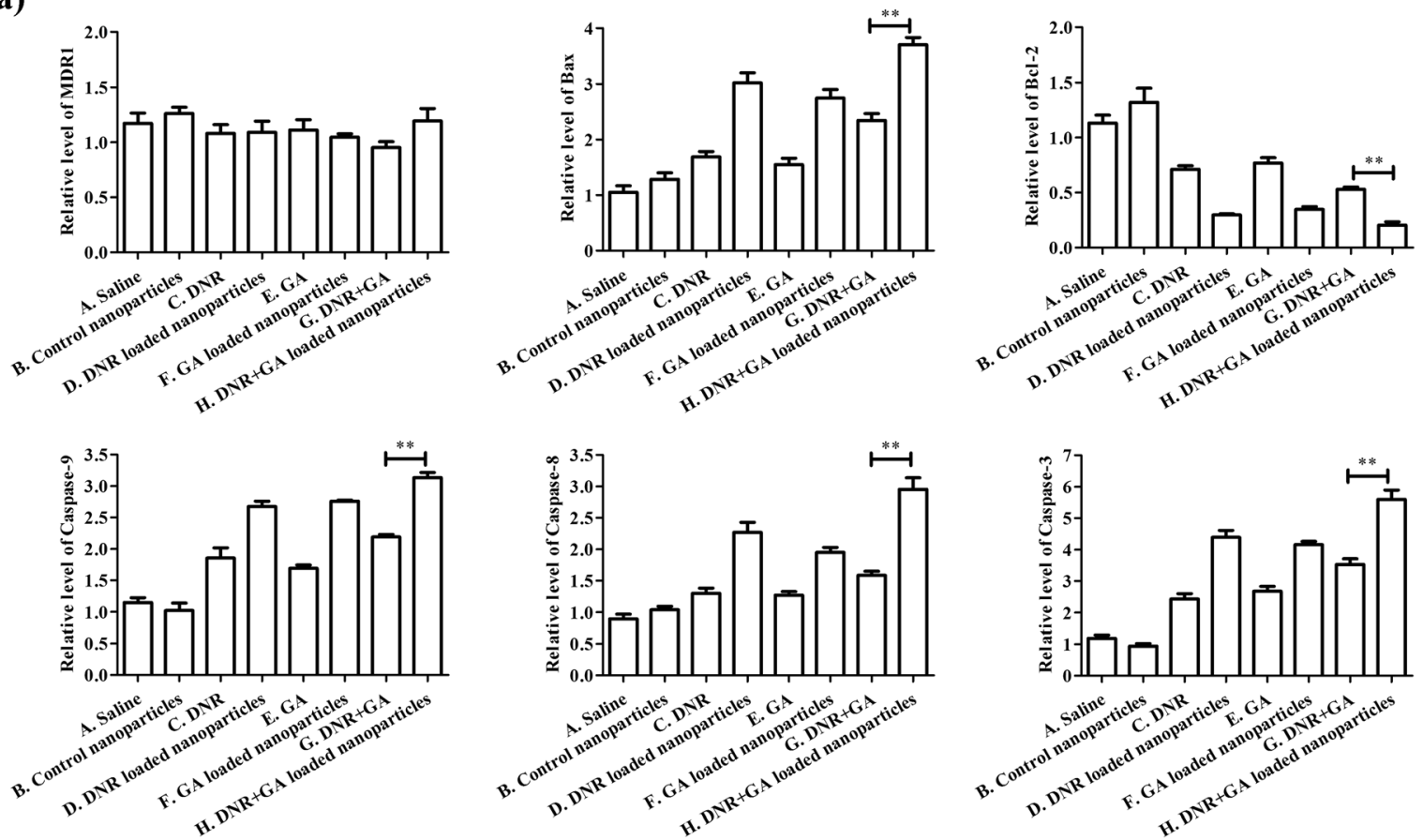

b)

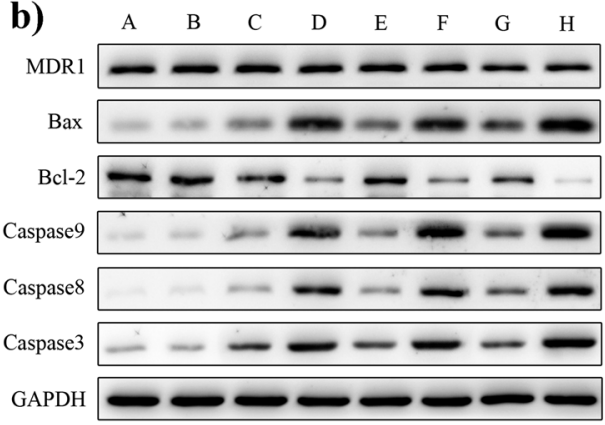

Fig. 7 a RT-PCR analysis of the expression of MDR1, the major gene related to drug resistance and Bax, Bcl-2, Caspase 9, Caspase 8 , and Caspase 3 , the genes associated with cell apoptosis. b Western blot analysis of the proteins corresponding to the genes;

change the therapeutic efficacy, neither in single treatment nor combined therapy.

In comparison, K562/A02 cells were strongly resistant to the treatment of free DNR or GA, as more than $60 \%$ of cancer cells survived the therapy after $24 \mathrm{~h}$. Dual drug therapy also failed to significantly reduce cell viability compared with that of a single drug. The poor antitumor effect is largely attributed to multidrug resistance of the cell. However, after being encapsulated into the nanosystem, the potency of each drug was significantly improved. The encapsulated DNR and GA achieved much enhanced cell inhibition compared to the non-encapsulated drugs, with approximately $50 \%$ of cancer cells eradicated. The most potent loss of cell viability was found in the group incubated with the dual drug-loaded nanoparticles with an inhibition ratio higher than $80 \%$, probably due to the synergistic effect of DNR and GA with different acting mechanisms. Therefore, nano-encapsulation could increase drug toxicity against the MDR leukemia cells to a very large extent, which was mainly attributed to the circumvention of drug resistance by nanoparticles. Of note, the effectiveness 
of the drug combination was only achieved after encapsulation, as evidenced by significantly improved cell toxicity compared to the simple blending $(P<0.01)$. Flow cytometry results were consistent with the MTT observation, and the number of apoptosis cells was the largest in the group treated with DNR/GA-loaded nanoparticles (Fig. 6). Thus, in the discovery of drug combinations in the treatment of drug-resistant leukemia, using nanocarriers can provide a more objective evaluation of the therapeutic efficacy that is overshadowed by MDR.

Mechanism of the apoptosis induced by DNR/ GA-loaded PVA/PLA nanoparticles

A major mechanism responsible for multiple drug resistance is the overexpression of the MDR1 gene, which encodes P-glycoprotein, an ATPase effluxing drug molecule out of the cell membrane. To explore how the nanosystem bypassed the resistance mechanism in K562/A02 cells, we evaluated the effects of different treatments on MDR1 and P-glycoprotein expressions. As shown in Fig. 7, the expressions of both MDR1 and P-glycoprotein were almost equal among the groups subjected to different treatments, and showed no significant difference compared with the saline control. Therefore, the enhanced therapeutic efficacy of the drug-loaded nanoparticles was not correlated with the regulation of MDR1 genes. Bcl-2 and $\mathrm{Bax}$ are responsible for the regulation of mitochondrial apoptosis induced by extracellular stresses and serve as anti-apoptotic and pro-apoptotic proteins, respectively. We then tested the expression of apoptosis-related genes and proteins by polymerase chain reaction and Western blot. K562/A02 cells treated with DNR + GA-loaded nanoparticles showed a significantly reduced expression of antiapoptotic protein (Bcl-2) compared with other groups $(P<0.01)$. On the other hand, the levels of activated Caspases 9, 8, and 3, the indicators of mitochondria-mediated apoptosis, were significantly increased in cells treated with the drugs encapsulated in nanoparticles compared to the free counterparts $(P<0.01)$. These observations pointed to the superior efficacy of our nano-preparations to regulate the apoptosis of the drug-resistant leukemia cells. As the MDR-related proteins were not influenced by our treatments, the greatly enhanced pro- apoptosis effect was most probably resulted from the targeted delivery of anticancer agents into cells, which increased intracellular drug concentration and bypassed the transmembrane effluxing mechanism. As in vivo toxicity is an important concern for further application of nanoparticles (Zhang et al. 2012), the toxicity of the nanoconstructs was tested in healthy mice. HE staining showed that free-drugs treatment caused obvious cardiotoxicity compared to the saline control, while the injection of the nanoparticles induced a negligible damage to normal organs including the heart, liver, spleen, lung, and kidney, which paved the way for future in vivo leukemia therapy.

\section{Conclusion}

Conclusively, we have developed a simple and versatile drug delivery strategy using PVA/PLA nanoparticles to circumvent the multiple drug resistance of leukemia cells. This system carries DNR and GA, a conventional broad-spectrum anticancer agent and a relatively new nature product recently proved effective in leukemia, respectively. With distinctly different mechanisms of action, the encapsulation of the two drugs in the nanoparticles has exhibited a remarkably enhanced therapeutic efficacy compared to the simple blending and singledrug treatments. Such observations suggest that the therapeutic potential of the drug combination can be fully unleashed by nano-encapsulation. The nanosystem did not alter the expression of the genes associated with drug resistance, but enhanced cell apoptosis by targeted drug delivery. Therefore, this strategy not only offers a potential strategy to bypass multidrug resistance by nano-encapsulation, but also inspires future design of new drug combinations using the biocompatible nanocarriers.

Authors' contributions HZ designed the experiments. LZ, YG, and XW performed the experiments and handed the data. PW wrote and edited the paper. PW and $\mathrm{HZ}$ contributed equally to this work.

Funding This project was supported by the National Natural Science Youth Foundation of China (No. 81601604), the Traditional Chinese Medicine of Jiangsu provincial administration (No. YB2015170), the Natural Science Youth Foundation of Jiangsu (No. BK20161070), the Cadre Health Research Project of Jiangsu 
Province (No. BJ15029), and Jiangsu Provincial Medical Youth Talent (No. QNRC2016531).

\section{Compliance with ethical standards}

Conflict interest The authors declare that they have no conflict of interest.

Open Access This article is distributed under the terms of the Creative Commons Attribution 4.0 International License (http:// creativecommons.org/licenses/by/4.0/), which permits unrestricted use, distribution, and reproduction in any medium, provided you give appropriate credit to the original author(s) and the source, provide a link to the Creative Commons license, and indicate if changes were made.

\section{References}

Bishop JF (1997) The treatment of adult acute myeloid leukemia. Semin Oncol 24:57-69

Cai Y, Xu Y, Chan HF, Fang X, He C, Chen M (2016) Glycyrrhetinic acid mediated drug delivery carriers for hepatocellular carcinoma therapy. Mol Pharm 13:699-709

Chen FY, Zhang Y, Chen XY, Li JQ, Xiao XP, Yu LL, Tang Q (2017) Development of a hybrid paclitaxel-loaded arsenite nanoparticle (HPAN) delivery system for synergistic combined therapy of paclitaxel-resistant cancer. J Nanopart Res 19:155

Csuk R (2014) Recent developments in the synthesis of antitumoractive glycyrrhetinic acid derivatives. Mini-Rev Org Chem $11: 253-261$

Cui F, Li Y, Zhou S, Jia M, Yang X, Yu F, Ye S, Hou Z, Xie L (2013) A comparative in vitro evaluation of self-assembled PTX-PLA and PTX-MPEG-PLA nanoparticles. Nanoscale Res Lett 8:301

Danafar H, Rostamizadeh K, Davaran S, Hamidi M (2014) PLAPEG-PLA copolymer-based polymersomes as nanocarriers for delivery of hydrophilic and hydrophobic drugs: preparation and evaluation with atorvastatin and lisinopril. Drug Dev Ind Pharm 40:1411-1420

Döhner H, Weisdorf DJ, Bloomfield CD (2015) Acute myeloid leukemi. N Engl J Med 373:1136-1152

Fox LC, Cummins KD, Costello B, Yeung D, Cleary R, Forsyth C, Tatarczuch M, Burbury K, Motorna O, Shortt J, Fleming S, McQuillan A, Schwarer A, Harrup R, Holmes A, Ratnasingam S, Chan KL, Hsu WH, Ashraf A, Putt F, Grigg A (2017) The incidence and natural history of dasatinib complications in the treatment of chronic myeloid leukemia. Blood Adv 1:802-811

Halley PD, Lucas CR, McWilliams EM, Webber MJ, Patton RA, Kural C, Lucas DM, Byrd JC, Castro CE (2016) Daunorubicin-loaded DNA origami nanostructures circumvent drug-resistance mechanisms in a leukemia model. Small $12: 308-320$
He SQ, Gao M, Fu YF et al (2015) Glycyrrhizic acid inhibits leukemia cell growth and migration via blocking AKT/ mTOR/STAT3 signaling. Int J Clin Exp Med 8:5175

Hibasami H, Iwase H, Yoshioka K, Takahashi H (2005) Glycyrrhizin induces apoptosis in human stomach cancer KATO III and human promyelotic leukemia HL-60 cells. Int J Mol Med 16:233-236

Holohan C, Van Schaeybroeck S, Longley DB et al (2013) Cancer drug resistance: an evolving paradigm. Nat Rev Cancer 13: 714-726

Kihara R, Nagata Y, Kiyoi H, Kato T, Yamamoto E, Suzuki K, Chen F, Asou N, Ohtake S, Miyawaki S, Miyazaki Y, Sakura T, Ozawa Y, Usui N, Kanamori H, Kiguchi T, Imai K, Uike N, Kimura F, Kitamura K, Nakaseko C, Onizuka M, Takeshita A, Ishida F, Suzushima H, Kato Y, Miwa H, Shiraishi Y, Chiba K, Tanaka H, Miyano S, Ogawa S, Naoe $\mathrm{T}$ (2014) Comprehensive analysis of genetic alterations and their prognostic impacts in adult acute myeloid leukemia patients. Leukemia 28:1586-1595

Kirtane AR, Kalscheuer SM, Panyam J (2013) Exploiting nanotechnology to overcome tumor drug resistance: challenges and opportunities. Adv Drug Deliv Rev 65:1731-1747

Li R, Wu R, Zhao L et al (2010) P-glycoprotein antibody functionalized carbon nanotube overcomes the multidrug resistance of human leukemia cells. ACS Nano 4:1399-1408

Li L, Osdal T, Ho Y, Chun S, McDonald T, Agarwal P, Lin A, Chu S, Qi J, Li L, Hsieh YT, Dos Santos C, Yuan H, Ha TQ, Popa M, Hovland R, Bruserud Ø, Gjertsen BT, Kuo YH, Chen W, Lain S, McCormack E, Bhatia R (2014) SIRT1 activation by a c-MYC oncogenic network promotes the maintenance and drug resistance of human FLT3-ITD acute myeloid leukemia stem cells. Cell Stem Cell 15:431-446

Loos C, Syrovets T, Musyanovych A, Mailänder V, Landfester K, Simmet T (2014) Amino-functionalized nanoparticles as inhibitors of mTOR and inducers of cell cycle arrest in leukemia cells. Biomaterials 35:1944-1953

Meng H, Mai WX, Zhang H, Xue M, Xia T, Lin S, Wang X, Zhao Y, Ji Z, Zink JI, Nel AE (2013) Codelivery of an optimal drug/siRNA combination using mesoporous silica nanoparticles to overcome drug resistance in breast cancer in vitro and in vivo. ACS Nano 7:994-1005

Mura S, Nicolas J, Couvreur P (2013) Stimuli-responsive nanocarriers for drug delivery. Nat Mater 12:991-1003

Nishida Y, Maeda A, Chachad D, Ishizawa J, Qiu YH, Kornblau SM, Kimura S, Andreeff M, Kojima K (2015) Preclinical activity of the novel B-cell-specific Moloney murine leukemia virus integration site 1 inhibitor PTC-209 in acute myeloid leukemia: implications for leukemia therapy. Cancer Sci 106:1705-1713

Picaud S, Fedorov O, Thanasopoulou A, Leonards K, Jones K, Meier J, Olzscha H, Monteiro O, Martin S, Philpott M, Tumber A, Filippakopoulos P, Yapp C, Wells C, Che KH, Bannister A, Robson S, Kumar U, Parr N, Lee K, Lugo D, Jeffrey P, Taylor S, Vecellio ML, Bountra C, Brennan PE, O'Mahony A, Velichko S, Muller S, Hay D, Daniels DL, Urh M, la Thangue NB, Kouzarides T, Prinjha R, Schwaller J, Knapp S (2015) Generation of a selective small molecule inhibitor of the $\mathrm{CBP} / \mathrm{p} 300$ bromodomain for leukemia therapy. Cancer Res 75:5106-5119 
Rochani AK, Girija AR, Borah A et al (2016) Heat-shock protein 90-targeted nano anticancer therapy. J Pharm Sci 105:14541466

Sheng X, Tucci J, Mittelman SD (2015) Reduction of oxidative stress promotes daunorubicin resistance in acute lymphoblastic leukemia cells. Cancer Res 75(15 Supplement):1247

Singh MR, Bart G, Zinke-Allmang M (2010) Polaron hopping in nano-scale poly (dA)-poly (dT) DNA. Nanoscale Res Lett 5: 501-504

Stauss HJ, Morris EC (2013) Immunotherapy with gene-modified T cells: limiting side effects provides new challenges. Gene Ther 20:1029-1032

Thirugnanam S, Xu L, Ramaswamy K, Gnanasekar M (2008) Glycyrrhizin induces apoptosis in prostate cancer cell lines DU-145 and LNCaP. Oncol Rep 20:1387-1392

Wang C, Xu H, Liang C, Liu Y, Li Z, Yang G, Cheng L, Li Y, Liu Z (2013) Iron oxide@ polypyrrole nanoparticles as a multifunctional drug carrier for remotely controlled cancer therapy with synergistic antitumor effect. ACS Nano 7:6782-6795

Whitlock JA, Greer JP, Lukens JN (1991) Epipodophyllotoxinrelated leukemia. Cancer 68:1-604

Zhang XY, Hu WB, Li J et al (2012) A comparative study of cellular uptake and cytotoxicity of multi-walled carbon nanotubes, graphene oxide, and nanodiamond. Toxicol Res 1:62-68

Zhang W, Gao C, Konopleva M, Chen Y, Jacamo RO, Borthakur G, Cortes JE, Ravandi F, Ramachandran A, Andreeff M
(2014) Reversal of acquired drug resistance in FLT3mutated acute myeloid leukemia cells via distinct drug combination strategies. Clin Cancer Res 20:2363-2374

Zhang Y, Chen H, Zhou S, Wang SX, Zheng K, Xu DD, Liu YT, Wang XY, Wang X, Yan HZ, Zhang L, Liu QY, Chen WQ, Wang YF (2015a) Sp1 and c-Myc modulate drug resistance of leukemia stem cells by regulating survivin expression through the ERK-MSK MAPK signaling pathway. Mol Cancer 14:56

Zhang XY, Wang K, Liu MY et al (2015b) Polymeric AIE-based nanoprobes for biomedical applications: recent advances and perspectives. Nanoscale 7:11486-11508

Zhao MX, Zhu BJ (2016) The research and applications of quantum dots as nano-carriers for targeted drug delivery and cancer therapy. Nanoscale Res Lett 11:207

Zhu H, Chen H, Zeng X, Wang Z, Zhang X, Wu Y, Gao Y, Zhang J, Liu K, Liu R, Cai L, Mei L, Feng SS (2014) Co-delivery of chemotherapeutic drugs with vitamin E TPGS by porous PLGA nanoparticles for enhanced chemotherapy against multi-drug resistance. Biomaterials 35:2391-2400

Publisher's note Springer Nature remains neutral with regard to jurisdictional claims in published maps and institutional affiliations. 